\title{
Insomnia and Neuroticism are Related with Depressive Symptoms of Medical Students
}

\author{
Changnam Kim, MD, Suyeon Lee, MD, Soyoung Youn, MD, Boram Park, MD, Seockhoon Chung, MD, PhD \\ Department of Psychiatry, Asan Medical Center, University of Ulsan College of Medicine, Seoul, Korea
}

\begin{abstract}
Background and Objective Insomnia is very common in depression and especially medical students are easy to experience sleep disturbance because of their studies. Also depressive symptoms are closely related to stress. Stress is an interaction between an individual and the environment, involving subjective perception and assessment of stressors, thus constituting a highly personalized process. Different personality traits can create different levels of stress. In this study, we tried to explore the relationship between insomnia and depressive symptoms or stress of medical students, and whether their personality may play a role on this relationship or not.

Methods We enrolled 154 medical students from University of Ulsan College of Medicine. We used the Medical Stress Scale, the Multidimensional Perfectionism Scale, the Patient Health Questionnaire-9, the Academic Motivation Scale, the Insomnia Severity Index, and The revised NEO Personality Inventory (PI).

Results Insomnia severity, amotivation, medical stress, mental health index and neuroticism traits of NEO-PI significantly correlated with depressive symptom severity $(\mathrm{p}<0.001)$. And stepwise linear regression analysis indicated insomnia, amotivation and neuroticism traits of NEO-PI are expecting factors for students' depressive symptoms is related to $(\mathrm{p}<0.001)$.

Conclusions Student who tend to be perfect feel more academic stress. The high level of depressive symptom is associated with insomnia, amotivation, academic stress in medical student. Moreover, personality trait also can influence their depressive symptoms.
\end{abstract}

Sleep Med Res 2017;8(1):33-38

Key Words Insomnia, Medical student, Academic stress, Depressive symptoms, Neuroticism.

Received: May 17, 2017

Revised: June 13, 2017

Accepted: June 19, 2017

Correspondence

Seockhoon Chung, MD, PhD

Department of Psychiatry,

Asan Medical Center, University of Ulsan

College of Medicine, 88 Olympic-ro 43-gil,

Songpa-gu, Seoul 05505, Korea

Tel $+82-2-3010-3411$

Fax +82-2-485-8381

E-mail schung@amc.seoul.kr

\section{INTRODUCTION}

Researchers have studied the condition of depression as manifest in medical students for several decades. Depressive symptoms are closely related to perceived stress. Since there are various type of stressors present during any period of formal medical training, ${ }^{1}$ medical students are readily susceptible to depressive symptoms. ${ }^{2}$ In recent study, $10 \%$ of medical students reported experiencing depressive symptoms, and majority of those individuals also reported suicidal ideation. ${ }^{3}$

Medical students are also prone to sleep disturbance. Their sleep-wake cycle is frequently interrupted by delayed sleep onset and inadequate sleep duration, with secondary daytime somnolence and napping. ${ }^{4}$ Their sleep deprivation might be related with lower academic performance. ${ }^{5}$ Low academic performance causes stress, which causes anxiety and other symptoms, which causes insomnia and sleep deprivation, and the sleep deprivation further degrades the academic performance causing further stress and more insomnia. This becomes a vicious cycle. ${ }^{6}$ However, the student's innate and/or acquired ability to assess and adapt to significant academic stress depends on the personality type and/or coping strategies of the particular individual. ${ }^{7}$ Stress is an interaction between the environment and the individual. Each person has his own personality, which includes subjective perception and coping strategies about stressors, so stress process is a highly personalized process. ${ }^{8}$

Previous studies showed that personality traits are associated with mood symptoms. High 
neuroticism was reported to be associated with major depressive disorder, and low extraversion was correlated with dysthymia in general population. ${ }^{9}$ Although psychological distress is a powerful predictor to depression among medical school students, their personality predispositions also affect depressive symptoms. ${ }^{10}$ High level of neuroticism is a strong risk factor of major depression in Swedish twin study. ${ }^{11}$ In Korea, medical students who have "avoidance" coping strategies tend to experience more stress than those who have other methods of coping with stress. ${ }^{12}$ In addition, insomnia or sleep disturbance also may be influenced by individual's personality. Harm avoidance and self-transcendence are positively related with insomnia. In contrast, novelty-seeking, reward dependence and cooperativeness are negatively related to severity of insomnia. Sleep quality is also correlated with self-transcendence. ${ }^{13}$

Therefore, it is important to explore whether the insomnia symptoms of medical students influences their medical stress or depressive symptoms. In this study, we tried to explore the relationship between insomnia and depressive symptoms or stress of medical students, and whether their personality may play a role on this relationship or not.

\section{METHODS}

\section{Participants}

We enrolled 154 medical students ranging from the second year of pre-medicine through the third year of medical school at the University of Ulsan College of Medicine, and assessed their current academic stress and psychological problems such as sleep problem or depressive symptoms in 2015.

Before starting of the survey, we explained all details about the aims and methods of the study to the 159 students. We distributed printed questionnaires to the students, and requested that the students fill in them. Finally data of 154 students, who have consented, were included in the final analyses. The official approval of this study was authorized by the Institutional Review Board of the Asan Medical Center.

\section{Measures}

Students' medical stress and academic motivation was assessed using the Medical Stress Scale (MSS) and Academic Motivation Scale (AMS). Their insomnia severity and depressive symptoms were checked using the Insomnia Severity Index (ISI) and Patient Health Questionnaire-9 (PHQ-9), Personality trait was assessed using the revised NEO Personality Inventory (NEO-PI) and the Multidimensional Perfectionism Scale (MPS).

The MSS is a self-report questionnaire to assess perceived stress levels in medical school, ${ }^{14}$ using a modified Korean version. The Korean version of the MSS consists of nine items focusing on three areas: school course of study and environment (four items); personal ability/perseverance (four items); and, and financial conditions (one item). ${ }^{15}$ Because all items had a 5 -point answer, a total score is 45 points. Thus we set the cutoff point as 28 for the present research.

The AMS consists of a 28-item questionnaire to assess academic motivation ${ }^{16}$ on the basis of self-determination theory ${ }^{17}$ and it has three subcategories such as intrinsic motivation, extrinsic motivation and amotivation. Higher score represent higher levels of motivation in each subcategory. We used the validated Korean AMS version in the present study. ${ }^{15}$

The ISI is a brief Self-Report Scale to evaluate the degree of both daytime and nighttime constituents of insomnia. The ISI consists of seven items to estimate the degree of sleep maintenance problems; sleep-onset difficulties and early morning awakening, satisfaction with his or her sleep state, influence on the functions of normal daytime life, severity of impairment of life of quality and level of concern caused by insomnia. All items have a $0-4$ scale so the range of total score is from 0 to 28 (0-7: normal, 8-14: mild insomnia, 15-21: moderate insomnia, 22-28: severe insomnia). ${ }^{18}$

The PHQ-9 is an instrument about the assessment of depressive symptoms based on DSM-IV criteria. It was developed as a self-report version of the original PRIME-MD by Spitzer et al. ${ }^{19}$ It consists of nine items about depressive symptoms, and there are one more question about functional impairment at the end of the diagnostic part of the PHQ-9: "How difficult have these problems made it for you to do your work, take care of things at home, or get along with other people?"20

The revised NEO-PI evaluates personality based on the big five model. It has 240 questions and consists of openness to experience, conscientiousness, extraversion, agreeableness and neuroticism. ${ }^{21}$

The MPS is a scale to assess the perfectionistic propensity. The MPS is invented by Frost et al. ${ }^{22}$ in 1990. It has six subscales (excessive concern over making mistakes, high personal standards, perception of high parental expectations, perception of high parental criticism, doubting of the quality of one's actions, and preference for order and organization) and 35 questions. Each item can be assessed using a 5-point Likert Scale, and total score can be calculated from 35 to 175 . As the score is higher, we consider that the student's perfectionism trait is stronger. In this study, the Korean version of MPS is used..$^{23}$

\section{Statistical Analysis}

A t-test of students has been used to make a comparison of the demographic and psychological characteristics between depressed students and non-depressed students. We also used Pearson's correlation analysis to determine the relationship between depressive symptoms and carious other psychological variables. Based on these results, stepwise linear regression analysis has been performed to find the factor related to depressive symptoms. We used The Statistical Package for the So- 
cial Sciences version 21.0 (SPSS Inc., Chicago, IL, USA) for all statistical analyses.

\section{RESULTS}

\section{Demographic and Psychological Characteristics of Participants}

In our study group, only 32 (20.7\%) students were female. Table 1 describes the demographic and psychological characteristics of the students grouped based on MSS score. In our participants, 29 (18.8\%) students have insomnia (ISI score $>7$ ). The ISI score is significantly high in stressed group $(\mathrm{p}<0.01)$ compared to non-stressed group. Also we found that stressed group has higher score about neuroticism, one aspect of NEOPI, than non-stressed group ( $\mathrm{p}<0.01)$. Statistically, there was no significant difference between the grades.

\section{Correlation of Depressive Symptoms with Psychological Characteristics}

We performed Pearson's correlation analysis to find out the association between depressive symptoms and other psychological characteristics (Table 2). ISI was found to be positively correlated with depressive symptom severity $(\mathrm{p}<0.01)$. Also, we have found that MSS and amotivation were positively correlated with depressive symptom severity $(\mathrm{p}<0.01)$. NEO-PImental health index and agreeableness trait were negatively correlated with PHQ-9 score $(\mathrm{p}<0.01)$. Neuroticism trait was positively correlated with PHQ-9 score $(\mathrm{p}<0.01)$.

Next, based on these findings we have constructed stepwise linear regression model (Table 3 ). High ISI score, amotivation, and neuroticism trait were predictor variables for depression. Because of statistical result, all explanatory variables are highly reliable.

\section{DISCUSSION}

We proved that stress is significantly related with insomnia. We also determined that medical students who have neuroticism trait experienced more stress in medical school. Furthermore, we found that students who have high level of depressive

Table 1. Demographic and psychological characteristics of participants

\begin{tabular}{|c|c|c|c|}
\hline Variables & Stressed $(n=55)$ & Non-stressed $(n=99)$ & p-value \\
\hline \multicolumn{4}{|l|}{ Gender } \\
\hline Male & 44 & 78 & \\
\hline Female & 11 & 21 & \\
\hline School year & $1.5 \pm 1.1$ & $1.5 \pm 1.1$ & 0.41 \\
\hline \multicolumn{4}{|l|}{ Motivation } \\
\hline Amotivation & $9.3 \pm 3.1$ & $6.9 \pm 2.4$ & 0.04 \\
\hline Intrinsic motivation & $38.9 \pm 7.2$ & $40.5 \pm 6.3$ & 0.30 \\
\hline Extrinsic motivation & $43.6 \pm 4.9$ & $43.4 \pm 5.5$ & 0.68 \\
\hline ISI & $6.1 \pm 4.2$ & $4.0 \pm 3.4$ & $<0.01$ \\
\hline PHQ-9 & $5.3 \pm 4.5$ & $2.7 \pm 2.7$ & 0.08 \\
\hline \multicolumn{4}{|l|}{ Perfectionism } \\
\hline Excessive concern over making mistakes & $26.6 \pm 6.5$ & $22.4 \pm 6.3$ & 0.99 \\
\hline High personal standards & $21.4 \pm 5.3$ & $20.3 \pm 5.1$ & 0.77 \\
\hline Perception of high parental expectations & $14.8 \pm 4.6$ & $13.7 \pm 4.5$ & 0.60 \\
\hline Perception of high parental criticism & $6.6 \pm 1.8$ & $5.6 \pm 2.1$ & 0.10 \\
\hline Doubting of the quality of one's actions & $11.6 \pm 3.6$ & $10.1 \pm 3.0$ & 0.12 \\
\hline Preference for order and organization & $18.3 \pm 5.6$ & $17.4 \pm 5.2$ & 0.48 \\
\hline NEO-PI-mental health index & $58.1 \pm 12.4$ & $68.0 \pm 10.8$ & 0.06 \\
\hline Extraversion & $43.8 \pm 10.3$ & $46.7 \pm 9.2$ & 0.56 \\
\hline Openness to experience & $47.8 \pm 10.0$ & $46.7 \pm 8.9$ & 0.61 \\
\hline Agreeableness & $41.5 \pm 8.4$ & $47.2 \pm 10.7$ & 0.15 \\
\hline Conscientiousness & $46.0 \pm 9.7$ & $47.3 \pm 8.3$ & 0.27 \\
\hline Neuroticism & $53.4 \pm 8.4$ & $46.5 \pm 6.3$ & $<0.01$ \\
\hline
\end{tabular}

'Stressed' students had Medical Stress Scale scores $\geq 28$ and 'non-stressed' students had MSS scores $<28$. 'School year' was re-coded as follows; 0: the second year of pre-medicine, 1: the first year of medicine, 2: the second year of medicine, 3: the third year of medicine.

ISI: Insomnia Severity Index, PHQ-9: Patient Health Questionnaire-9, NEO-PI: NEO Personality Inventory, MSS: Medical Stress Scale. 
symptoms tend to experience more insomnia and loss of motivation. Stress has a cause-and-effect relationship with depression, as shown by numerous previous studies. It became clear that chronic exposure to perceived stressors caused depressionlike stress by making molecular, cellular, and behavioral changes. ${ }^{24}$ Personality traits might also influence the severity of the depressive symptoms present. In stepwise linear regression model high ISI score, loss of motivation and neuroticism trait is a risk factor of symptom severity of depression of the students. Overall, insomnia and neuroticism trait of NEO-PI index are powerful factors related to experience depressive symptoms. In addition, motivation is importantly correlated with depression. According to our research, medical students who have sufficient and substantial motivation tend to experience less stress and fewer depressive symptoms.

Insomnia is closely linked to enrollment in medical school.

Table 2. Correlation of depressive symptoms with psychological characteristics

\begin{tabular}{lcc}
\hline \multicolumn{1}{c}{ Variables } & Pearson's $\mathrm{R}^{2}$ & $\mathrm{p}$-value \\
\hline Motivation & & \\
Amotivation & 0.30 & $<0.01$ \\
Intrinsic motivation & -0.19 & 0.02 \\
Extrinsic motivation & -0.12 & 0.15 \\
ISI & 0.31 & $<0.01$ \\
MSS & 0.31 & $<0.01$ \\
Perfectionism & & \\
Excessive concern over making & 0.25 & 0.002 \\
$\quad$ mistakes & & \\
High personal standards & 0.01 & 0.92 \\
Perception of high parental & 0.09 & 0.30 \\
$\quad$ expectations & & \\
Perception of high parental criticism & 0.16 & 0.04 \\
Doubting of the quality of one's actions & 0.20 & 0.01 \\
Preference for order and organization & -0.08 & 0.30 \\
NEO-PI-mental health index & -0.40 & $<0.01$ \\
Extraversion & -0.15 & 0.07 \\
Openness to experience & 0.02 & 0.78 \\
Agreeableness & -0.26 & 0.001 \\
Conscientiousness & -0.17 & 0.04 \\
Neuroticism & 0.42 & $<0.01$ \\
\hline
\end{tabular}

NEO-PI: NEO Personality Inventory, ISI: Insomnia Severity Index, MSS: Medical Stress Scale.
There are already a proliferation of studies addressing the relationship between medical school, medical students and sleep disorders. In Asia, numerous researches were done to assess insomnia severity in medical students in China, Hong Kong, Malaysia, India, and Iran. In North and South America, United States, Brazil, and Mexico have epidemiological data on insomnia of medical students. ${ }^{25}$ The attitudes, academic load, life style choices, potentially sleep apnea and internet usage may induce or exacerbate sleep disturbance. ${ }^{25}$ Therefore, insomnia in medical students is considered a very important problem, worldwide.

Prior literary sources indicate document the belief that various psychiatric disorders have higher incidence and prevalence when the afflicted individuals suffer from insomnia. Insomnia patients are about four times more likely to develop depression than those who sleep well. ${ }^{26}$ Besides, there are some research efforts showing that sleep problems would be a predictive factor of psychiatric disorder like alcohol abuse, anxiety or depression. ${ }^{26,27}$ In addition, some researchers performed several studies about subgroups of insomniacs in past years. They prove that women, shift workers and those who simply don't sleep enough are more vulnerable to insomnia. ${ }^{28}$ As it has been concluded by previous researchers, our study reveals that insomnia is correlates significantly with depression. So if a medical student visits your office complains about sleep disturbance, it might be best if you look more closely and assess for other problems like symptoms of depression.

Our research indicates that some personality traits are related to depressive symptoms, too. Over previous decades, many researchers published papers documenting the possible relationship between personality and psychiatric disorders. In general population, the patient diagnosed with anxiety and depressive disorders will likely have more extraversion and neuroticism traits. ${ }^{10}$ In meta-analysis including 175 studies, it was trait of "neuroticism" which was the most obviously and most often linked to the full spectrum of psychiatric disorders, and some other traits had considerable correlation apart from neuroticism. ${ }^{29}$ In our study, the participants are all medical students, and some part of results was in line with the previous literatures.

Neuroticism is the propensity to experience pessimistic emotions, for example anxiety or sadness, including mood swings, ${ }^{30}$ and has been regarded as one of the personality traits which is related to psychopathology, especially depression and anxiety. ${ }^{31}$ Researchers have focused on identifying potential the

Table 3. Result of stepwise linear regression (B) for expecting depressive symptoms measured using PHQ-9

\begin{tabular}{ccccc}
\hline Outcome variables & Explanatory variables & $\beta$ & $\mathrm{B}$ & $\mathrm{p}$-value \\
\hline PHQ-9 $\left(\mathrm{R}^{2}=0.455, \mathrm{p}<0.001\right)$ & ISI & 0.39 & 0.37 & $<0.01$ \\
& Amotivation & 0.22 & 0.28 & $<0.01$ \\
& Neuroticism & 0.34 & 0.16 & $<0.01$ \\
\hline
\end{tabular}

ISI: Insomnia Severity Index, PHQ-9: Patient Health Questionnaire-9. 
biological evidence linking neuroticism and mood symptoms. Some of them proved that neuroticism indicated quantitative trait loci on chromosomes 1, 4, 7, 12 and $13^{32}$ and another group of scientists revealed that serotonin transporter and gammaaminobutyric acid(A) alpha 6 receptor variants are correlated with neuroticism. ${ }^{33}$ However and to date, evidence of any biological basis to suggest, or indicate, a relationship between neuroticism and depression remains unclear (and controversial).

Motivation has been shown to affect the depressive symptoms in medical students. In medical school, there are various type of "stress" including "academic stress" ${ }^{34}$ When one considers the competitive climate which characterizes traditional medical school, it would be reasonable to assume that the medical students experience a substantial amount of academic stress. Thus, we might be well-served to consider establishment of a plan designed to decrease academic pressure to enhance motivation. ${ }^{35}$ Furthermore, various types of approaches are needed to improve academic motivation, including social cognitive theory such as reinforce self-efficacy belief. ${ }^{36}$ In present study, we also tested the partial link between the depressive symptoms and the trait of "perfectionism", and a tendency toward self-doubt, the quality or correctness of one's actions in retrospect. If necessary, a mal-adaptive "perfectionism" trait might be corrected for the sake of the mental health of the medical student in question.

There are several constraints in play in the current study. First of all, we had no means of verifying any of the participants' self-described medical histories, especially when it came to the area of psychiatrics. If there was to be a large or disproportionate percentage of depressive disorder among our participants, it could adversely affect the raw data and the results. Second and as noted, this research is based on self-report questionnaires which contain subjective bias. However, the most of participants have experience to filling out questionnaires much like hours at some point during the last year. So they would be appropriately familiar with that process. Third, this was a crosssectional study. So we could find the association between variables, but it was impossible to establish the causality. Forth, we didn't accomplish the comparison about medical students and the students who have another major. In Korea, college students are in the most stressful situation including seeking a full-time job and student loan repayment. So the influence of insomnia and stress could be different to non-medical students. Furthermore there would be a personality difference between them. Further studies will be performed considering the possibilities.

\section{Conflicts of Interest}

The authors have no financial conflicts of interest.

\section{REFERENCES}

1. Levey RE. Sources of stress for residents and recommendations for programs to assist them. Acad Med 2001;76:142-50.

2. Mosley TH Jr, Perrin SG, Neral SM, Dubbert PM, Grothues CA, Pinto BM. Stress, coping, and well-being among third-year medical students. Acad Med 1994;69:765-7.

3. Goebert D, Thompson D, Takeshita J, Beach C, Bryson P, Ephgrave K, et al. Depressive symptoms in medical students and residents: a multischool study. Acad Med 2009;84:236-41.

4. Abdulghani HM, Alrowais NA, Bin-Saad NS, Al-Subaie NM, Haji AM, Alhaqwi AI. Sleep disorder among medical students: relationship to their academic performance. Med Teach 2012;34 Suppl 1:S37-41.

5. Medeiros ALD, Mendes DB, Lima PF, Araujo JF. The relationships between sleep-wake cycle and academic performance in medical students. Biol Rhythm Res 2001;32:263-70.

6. Akerstedt T. Psychosocial stress and impaired sleep. Scand J Work Environ Health 2006;32:493-501.

7. van Berkel HK. The relationship between personality, coping styles and stress, anxiety and depression [dissertation]. Christchurch: University of Canterbury 2009.

8. Zeidner M, Endler NS. Handbook of coping: theory, research, applications. New York: John Wiley \& Sons 1996.

9. Bienvenu OJ, Samuels JF, Costa PT, Reti IM, Eaton WW, Nestadt G. Anxiety and depressive disorders and the five-factor model of personality: a higher- and lower-order personality trait investigation in a community sample. Depress Anxiety 2004;20:92-7.

10. Matsudaira T, Kitamura T. Personality traits as risk factors of depression and anxiety among Japanese students. J Clin Psychol 2006;62:97109.

11. Kendler KS, Gatz M, Gardner CO, Pedersen NL. Personality and major depression: a Swedish longitudinal, population-based twin study. Arch Gen Psychiatry 2006;63:1113-20.

12. An H, Chung S, Park J, Kim SY, Kim KM, Kim KS. Novelty-seeking and avoidant coping strategies are associated with academic stress in Korean medical students. Psychiatry Res 2012;200:464-8.

13. Park JH, An H, Jang ES, Chung S. The influence of personality and dysfunctional sleep-related cognitions on the severity of insomnia. Psychiatry Res 2012;197:275-9.

14. Vitaliano PP, Maiuro RD, Mitchell E, Russo J. Perceived stress in medical school: resistors, persistors, adaptors and maladaptors. Soc Sci Med 1989;28:1321-9.

15. Ahn D, Park G, Baek KJ, Chung SI. Academic motivation, academic stress, and perceptions of academic performance in medical students. Korean J Med Educ 2007;19:59-71.

16. Vallerand RJ, Pelletier LG, Blais MR, Briere NM, Senecal C, Vallieres EF. The Academic Motivation Scale: a measure of intrinsic, extrinsic, and amotivation in education. Educ Psychol Meas 1992;52:1003-17.

17. Fairchild AJ, Horst SJ, Finney SJ, Barron KE. Evaluating existing and new validity evidence for the Academic Motivation Scale. Contemp Educ Psychol 2005;30:331-58.

18. Bastien $\mathrm{CH}$, Vallières A, Morin CM. Validation of the Insomnia Severity Index as an outcome measure for insomnia research. Sleep Med 2001;2:297-307.

19. Spitzer RL, Kroenke K, Williams JB. Validation and utility of a self-report version of PRIME-MD: the PHQ primary care study. Primary care evaluation of mental disorders. Patient Health Questionnaire. JAMA 1999;282:1737-44.

20. Kroenke K, Spitzer RL. The PHQ-9: a new depression diagnostic and severity measure. Psychiatr Ann 2002;32:509-15.

21. Costa PT, McCrae RR. Normal personality assessment in clinical practice: the NEO Personality Inventory. Psychol Assess 1992;4:5.

22. Frost RO, Marten P, Lahart C, Rosenblate R. The dimensions of perfectionism. Cogn Ther Res 1990;14:449-68.

23. Han K. Multidimensional perfectionism: concept, measurement and relevance to maladjustment [dissertation]. Seoul: Korea University 1993.

24. Pittenger C, Duman RS. Stress, depression, and neuroplasticity: a convergence of mechanisms. Neuropsychopharmacology 2008;33:88-109. 
25. Azad MC, Fraser K, Rumana N, Abdullah AF, Shahana N, Hanly PJ, et al. Sleep disturbances among medical students: a global perspective. $J$ Clin Sleep Med 2015;11:69-74.

26. Ford DE, Kamerow DB. Epidemiologic study of sleep disturbances and psychiatric disorders. An opportunity for prevention? JAMA 1989;262:1479-84.

27. Ohayon MM, Caulet M, Priest RG, Guilleminault C. DSM-IV and ICSD-90 insomnia symptoms and sleep dissatisfaction. Br J Psychiatry 1997;171:382-8.

28. Häkkänen H, Summala H. Sleepiness at work among commercial truck drivers. Sleep 2000;23:49-57.

29. Kotov R, Gamez W, Schmidt F, Watson D. Linking "big" personality traits to anxiety, depressive, and substance use disorders: a meta-analysis. Psychol Bull 2010;136:768-821.

30. Costa PT, McCrae RR. The NEO Personality Inventory: manual form $S$ and form R. Odessa, FL: Psychological Assessment Resources 1985.

31. Roelofs J, Huibers M, Peeters F, Arntz A, van Os J. Rumination and worrying as possible mediators in the relation between neuroticism and symptoms of depression and anxiety in clinically depressed individuals. Behav Res Ther 2008;46:1283-9.

32. Nash MW, Huezo-Diaz P, Williamson RJ, Sterne A, Purcell S, Hoda F, et al. Genome-wide linkage analysis of a composite index of neuroticism and mood-related scales in extreme selected sibships. Hum Mol Genet 2004;13:2173-82.

33. Sen S, Villafuerte S, Nesse R, Stoltenberg SF, Hopcian J, Gleiberman L, et al. Serotonin transporter and GABAA alpha 6 receptor variants are associated with neuroticism. Biol Psychiatry 2004;55:244-9.

34. Stewart SM, Lam TH, Betson CL, Wong CM, Wong AM. A prospective analysis of stress and academic performance in the first two years of medical school. Med Educ 1999;33:243-50.

35. Park J, Chung S, An H, Park S, Lee C, Kim SY, et al. A structural model of stress, motivation, and academic performance in medical students. Psychiatry Investig 2012;9:143-9.

36. Zimmerman BJ, Bandura A, Martinez-Pons M. Self-motivation for academic attainment: the role of self-efficacy beliefs and personal goal setting. Am Educ Res J 1992;29:663-76. 